mechanism is capable of putting the spine into a distorted position with curves in opposite directions, but not of causing bony rotation on the convex side of the lateral curve.

The occurrence of such bony rotation must be regarded as a superadded pathological change in structure due to abnormal plasticity of bone in the affected individual.

Up to the point of bony rotation one must remember that the phenomena can be experimentally reproduced in any normal cadaver, model or child, but that bony rotation cannot be experimentally reproduced and cannot be explained in terms of spinal movements.

I would, therefore, ask you to regard concavesided rotation as in a measure physiological and convex-sided rotation as pathological, not that the two phenomena are sharply separated, for, of course, they run into each other and overlap, but for purposes of greater clearness the types of the two should, I think, be kept separate.

Bony rotation. - The question of the cause of bony rotation lies outside of my present line of inquiry, and has been amply treated by German writers. ${ }^{17}$ It seems to me to be explained by the fact that the vertebræ of some children are plastic and ready to be distorted by increased pressure on one side and diminished pressure on the other, and that Wullstein's experiments on the dogs have proved this, if we need proof. In a column curved to one side or to both sides in different portions the pressure is greatest on the side of the vertebra next to the concavity of the curve and least on the side of the convexity, because the line of weight falls to the median side of the concavity. If the vertebræ are plastic, they will yield in the line of least resistance; that is, toward the side of the convexity of the lateral curve.

A plastic weight bearing column curved in two directions will not yield straight to the side, but will also twist. The vertebral column in all parts is curved in the anteroposterior plane by the physiological curves. The rotation will naturally be away from the line of gravity, the median line of the column, because to twist toward it would be to encounter the greatest instead of the least resistance. It seems to me, therefore, wholly in accord with what we know of general mechanics that in a plastic weightbearing column curved in two planes bony rotation must occur on the convex side of each lateral curve.

Classification. - From this point of view, there are then two distinct divisions of lateral curvature, - one, the total curves accompanied by prominence of the back on the concave side of the lateral curve (rotation on the right, in curves convex to the left) which are to be regarded as postural and not accompanied by extensive pathological changes.

The other, the compound or more rarely, single curves accompanied by prominence of the back on the convex side of the lateral curve (rota-

17 Riedinger: Morph. und Mech. d. Skol., Wiesbaden, 1901.

Schanz: Die Belast. Def. d. Wirkelsäule, Stuttgart, 1904.

Hoffa: Orth. Chir., Sth ed., p. 328. tion on the left, in curves convex to the left and vice versa). These represent the cases accompanied by pathological changes in the vertebræ, Finally transition cases are to be recognized.

Summary. - No summary is possible of a paper which in its entirety is only a summing up of certain facts in a field of study large enough to fill a volume. The points that I have tried to establish from anatomical and clinical data are that total postural scoliosis is to be regarded as as within physiological limits and that it can be accounted for by the normal mechanism of the spine; that the same normal mechanism will account for the production of compound curves from simple ones, but that it will not account for the occurrence of bony rotation on the convex side of the lateral curve which must be regarded as a superadded pathological change. Certain of the anatomical facts considered should be of direct use in making more exact the gymnastic treatment of slight scoliosis, but that they can be carried over into the gymnastic treatment of structural scoliosis without very careful consideration in each case does not seem to me likely. In the latter case we are no longer dealing with a spine distorted by the normal mechanism, but by a malposition to which is added a distorting pathologic process. That a knowledge of spinal mechanics will make clearer the treatment of structural scoliosis I have no question, but the application of this knowledge will require further study and careful investigation.

\section{FIRST OF A SERIES OF ARTICLES ON THE APPENDIX VERMIFORMIS.}

\footnotetext{
BY WM. A. BROOR3, JR., A.M., M.D., BOsTON, - Assistant Surgeon, Massachusetts General Hospital.
}

Position. - In tracing the development of the human embryo, the appendix vermiformis is first seen immediately following the differentiation of the large and small intestine. It is then found high up on the right side of the abdomen, close to the liver.

As the ascending colon develops and obtains its growth downwards, the cecum with the appendix occupies a lower and lower position on the right side of the abdomen, until, with the full development, the cecum and its appendix come to rest in the right iliac fossa, and it is here that the appendix is found in a great majority of cases. Frequently only the base of the appendix rests in the right iliac fossa, while the remainder lies towards the median line or hangs over into the true pelvis.

The longitudinal bands on the ascending colon and cecum invariably lead downwards to the base of the appendix.

With the fastening back of the ascending colon owing to the gluing together of the posterior visceral layer covering the ascending colon and its mesentery and the parietal peritoneum lining the posterior wall of the abdominal cavity, the cecum and its appendix are practically held in one position, and the excursions of the appendix 
are limited by the degree of freedom of the cecum and the length of its own mesentery.

It is in these normal cases that the McBurney point is of value, and in these cases alone.

Abnormal positions. - If, for any reason, the development of the ascending colon is arrested it will be easily understood why the appendix may be found at any point between the right iliac fossa and the liver.

Should the ascending colon fail to become attached to the posterior wall, the appendix may be found almost anywhere within the abdominal cavity.

Example. - There is a subject of a sixteen-y earold girl at the Harvard Medical School which presents just this condition of affairs. The ascending colon is completely unattached to the posterior abdominal wall except by the root of its mesentery. The appendix may be placed at almost any point in the abdominal cavity, within the radius of its own mesentery and the mesentery of the ascending colon.

Causes of acute appendicitis. - In a general way it may be said that any thing which forcibly obstructs the lumen of the appendix or anything which seriously interferes with the circulation of the appendix, will bring about an attack. In a great majority of cases fecal concretions are found to have been the cause of an attack, and it can be readily understood how, as a result of peristaltic action, fecal matter is forced into the appendix and becoming more and more firmly wedged towards the tip, affects the circulation in the wall and leads to obstruction, ulceration and perforation or gangrene. A kink or twist in the organ may produce the same result.

For a time it was considered that the presence of a foreign body was the most common cause. Examination of hundreds of cases has shown this is not the case.

It is a question whether some cases of acute appendicitis seen in elderly people are not indirectly due to arterial changes, a slight accumulation of fecal matter producing a result which would not be expected if the organ was well nourished.

A great deal has been written about catarrhal inflammation of the cecum and the ascending colon extending into the appendix. These cases are more apt to be chronic and do not, as a rule, present the serious acute attack.

Infrequently there are seen cases which are the result of a sudden perforation of a chronic ulcer, the ulcer being part of the process that is going on within the cecum and the ascending colon.

To sum it all up, the severe acute attack of appendicitis is generally due to an occlusion of the lumen or of a shutting off of the blood supply from some portion or greater part of the appendix itself.

Note. - It has been observed that appendices whose mesenteries do not extend well to the tip are more liable to become gangrenous when their lumens are occluded. Those appendices with complete mesenteries are more subject to the suppurative type of the disease,
Symptoms. -- The group of symptoms which any one patient presents when suffering from an acute attack of appendicitis is not sufficiently reliable to make a diagnosis from for all cases.

The sudden onset, pain, vomiting, tenderness, rigidity of the abdominal muscles, high pulse and temperature may or may not be noted in any one case.

As a rule, for some unknown reason, the attack begins at night. The patient wakes up suffering from abdominal pain. This pain may be general in character. It may or may not be accompanied by vomiting. There may or may not be an elevation of pulse and temperature. If the inflamed appendix is so situated that pressure can be put upon it, tenderness can always be found.

When, however, the appendix lies over in the true pelvis, so situated that pressure either manually or bi-manually cannot be put upon it, even this symptom of tenderness cannot be elicited.

If such a case is allowed to progress until most of the cardinal symptoms present themselves, the diagnosis is no longer in doubt. But while waiting for these symptoms to become established in order to make a definite diagnosis, the danger to the patient is increased many-fold.

Some authorities state that in every case of appendicitis they expect to find four symptoms: localized pain, tenderness, elevation of pulse and temperature, nausea and vomiting. Experience has shown that the symptom of tenderness is by far the most reliable and the most constant. Other symptoms vary so markedly and are so many times found wanting that they are not reliable. If not found they should not be waited for.

The previous history of a patient may be of value. There may be a history of previous attacks of abdominal disturbances or of welldefined attacks of appendicitis. Chronic constipation has a distinct bearing. Attacks of abdominal pain and diarrhea are to be considered. In fully $50 \%$ of the cases the previous history is negative.

When to operate. - There is no question about the advisability of operating upon every case where the appendix is suspected and where within a few hours after the initial symptom or symptoms, a rapid improvement has not taken place.

Exception. - Cases in which complication of other diseases makes the operative risk too great.

One of Boston's best surgeons once remarked that the time to operate upon a case of appendicitis was before the diagnosis was made. This sounds absurd, but it appeals to many surgeons who have been called too late many times and have been beaten in the struggle to save the life of the patient.

Waiting until every symptom is present and the diagnosis is beyond dispute means taking away from the patient many chances of recovery.

Probably there is no affection which presents so many and so varied conditions within a comparatively short space of time. Other diseases run their course and we know something about 
what to expect as the hours and days go by, but no one can tell the condition of the appendix by judging from the symptoms which the case presents.

Perforation may take place with the initial pain and general peritonitis may set in almost immediately. The appendix may become gangrenous within a few hours. The only positive way of knowing what the condition is, is by opening the abdomen and inspecting the appendix.

Without question many cases of appendicitis have been cured by medical treatment and many others will be. It is also true that many cases have been lost by being treated medically or by waiting for the diagnosis to be thoroughly established.

It is a well-known fact that the patient after having one attack of appendicitis is liable to a recurrence of the trouble at any time, and the subsequent attack may be fatal. These facts force one to the conclusion that it is better to operate and remove the seat of the trouble, than to congratulate oneself on a cure which leaves the patient forever liable to another attack.

The operation for acute appendicitis. - Preparation of the patient: While the preparation of the patient must be quickly performed it must be done thoroughly. If necessary the patient is catheterized. No attempt is made to evacuate the bowels.

The lower part of the body must be shaved. After a thorough scrubbing with soap and water, care being taken not to excoriate the skin, the soap is removed by allowing a solution of corrosive sublimate, 1-3000, to drip from pieces of sterile gauze. The part is then wiped by ether followed by alcohol. As soon as the patient is under the influence of an anesthetic, the abdomen is again gently scrubbed and sterile towels placed about.

The incision. - Formerly it was considered that the incision should be an oblique one, about half way between the anterior superior spine and the umbilicus, running parallel with Poupart's ligament. This incision is practically obsolete.

The majority of surgeons now prefer either to go through the rectus muscle on the right and open through into the abdominal cavity; or, having made an incision down to the rectus, to retract that muscle towards the median line and then go through into the abdominal cavity.

As in every case of laparotomy great care must be taken not to injure the intestines when the peritoneum is opened.

The gridiron incision should never be used in operating for an attack of acute appendicitis. As one never can tell what the condition of the appendix is, there is danger in an incision which cannot be enlarged without serious damage to the parts.

Technique. - When the abdominal cavity is opened, many men are in the habit of introducing the fingers in order to determine the location and condition of the appendix. Such a procedure has its dangers. Slight adhesions may be broken up and septic material scattered about in the abdominal cavity.
By far the best method is the careful introduction of walling-off gauze, above to the inner side and below the region of the appendix.

Having placed these safe-guards, by the introduction of a retractor, the outer edge of the wound can be drawn outwards and the cecum brought into view. The cecum can then be drawn up into the wound, and if it is comparatively free, the affected appendix can be brought completely outside of the abdominal cavity. Gauze can then be packed around the cecum; the mesentery of the appendix tied and cut away; the appendix itself securely tied at its base after its contents have been stripped either back into the cecum or towards the tip of the appendix by the gentle pressure of a hemostat. A hemostat is firmly closed on the appendix, so placed as to leave sufficient space between the ligature around the base of the appendix and the hemostat for the appendix to be severed with the actual cautery.

The custom of cutting through the appendix with a knife or scissors and the application of $95 \%$ solution of carbolic acid to the stump is not so satisfactory a procedure as is the use of the actual cautery, for the reason that it is difficult to absolutely control the caustic effects of the acid.

After the appendix has been removed one of two methods may be employed. Either the cecum with the cauterized stump of the appendix may be dropped back into the abdominal cavity, or by means of a pursestring suture taken in the head of the cecum, the stump may be covered in, the stump of the appendix being pushed towards the cecum as the suture is drawn tight. Any spaces between the peritoneal surfaces are then closed by fine intestinal sutures.

While some consider that the use of the pursestring suture has its danger from the fact that the needle may be allowed to penetrate into the intestine, if the suture is applied carefully this danger does not exist. The patient suffers much less pain and discomfort when the stump is covered in, and there is much less liability of the development of a fecal fistula, either as a result of subsequent sloughing of the stump of the appendix, or by the slipping of the ligature.

In a certain number of cases in which, subsequent to the operation, the large intestine has become distended with gas, the stump of the appendix has drawn out from the ligature. This fact would suggest the advisability of transfixing the stump if it is not going to be covered in. The cecum and the stump of the appendix having been returned to the abdominal cavity, the gauze packing is removed. A small wick is left extending down to the base of the appendix. The remainder of the wound is closed by interrupted through and through sutures or by continuous sutures of the different layers.

The day has passed when the surgeon, relying on his judgment of the conditions which he can see in the neighborhood of an acutely inflamed appendix, completely closes the abdominal cavity. The leaving of a small wick is a practical guarantee 
of the safety of the patient, and if absorbable ligatures have been used there is no danger of a persistant sinus.

After care. - The after care of a patient who has been operated upon for an uncomplicated attack of acute appendicitis is a simple matter. As soon as the stomach has quieted down from the effects of the anesthetic, sips of hot or cold water may be given. The patient should be kept quiet and made to lie on his back. After the first twenty-four hours an attempt may be made to move the bowels by enemata and at the end of forty-eight hours small doses of calomel, 1 10 $\mathrm{gr}$. each, can be given every hour, for eight or ten doses, to be followed by a saline the next morning.

At the end of twenty-four hours or as soon as the bowels have moved, the patient can be given liquids in any form. If these are well borne, they can be followed by soft solids, and then by solids, so that at the end of three days the patient is practically on a regular diet.

The gauze wick, if the temperature and pulse are favorable, can be started at the end of twentyfour hours, shortened the following day or removed entirely. After the removal of the wick, the edges of the wound can be drawn together by a strip of adhesive plaster. At the end of a week the patient can be allowed to sit up in bed and at the end of ten days it is safe to have the patient sit up out of bed. In from twelve to fourteen days the patient may be allowed to walk about.

\section{Elinical dDepartment.}

\section{THE EARLY DIAGNOSIS OF MALIGNANT DIS- EASE OF THE STOMACH AND INTESTINES.*}

BY WILLIAM R. ROBEY, JR., M.D.,

Physician to Out-Patients at the Boston City Hospital; Assistant in Clinical Medicine, Harvard Medical School.

THE study of cases of abdominal disease is of steadily increasing interest. I use the broad term of abdominal disease because when the case is first seen we are often at a loss to tell the specific organ involved. These cases are of great interest to the surgeon but they are of greater interest to the stomach specialist and physician because with him rests the decision of what shall be done in the individual case. The surgeon complains because the patient is brought to him by the physician too late to make his operation anything but a last resort. The conscientious physician is striving not to have it so. $\mathrm{He}$ retains a case merely because he honestly does not know at what stage in the disease to offer operation to his patient. The reason for this is because our present methods of stomach examination are so uncertain in pointing to definite conditions and because many of the malignant cases are so well advanced before they offer themselves for treatment.

Dr. J. C. Munro says that the majority of patients seeking aid of the medical practitioner and the stomach specialist are medical and not

\footnotetext{
* Read before Norfolk District Medical Society, Feb. 28, 1905.
}

surgical from first to last. These are the dyspeptics from alcohol, dietary errors, worry, etc., and they can all be cured far better in any way than by surgery.

We are accepting surgery for relapsing ulcer, hemorrhage and, of course, for perforating ulcer. In relapsing ulcer we have more time for diagnosis. A simple ulcer will yield to the ordinary medicinal measures and may not recur, but where there are repeated relapses with distress, pain and hemorrhages we believe now that surgical interference offers the best if not the only outcome for the case. If the case is treated medically the patient should be told it will require at least three months of strict attention. After recovery there should be comparatively good health, and if not, that is, if there is indigestion, epigastric pain and vomiting, it is clearly the duty of the physician to call the surgeon in consultation with a view to warding off the danger of hemorrhage or perforation or, if not these serious accidents, continued ill health with the possible malignant growth on the site of the old ulcer. As some of our cases of malignant disease show very few striking symptoms before the patient is past all help, so in ulcer the first intimation of its presence is hemorrhage or perforation. Osler says that a fatal result is not very common from hemorrhage, but the recent review of Lund, Joslin and Murphy shows a high mortality from this accident. They furthermore show that $56 \%$ of the ulcer cases medically treated were either dead or had stomach trouble after their discharge from the hospital.

In private practice the good results from medical treatment are more readily maintained.

It is necessary for the physician to keep in touch with his stomach cases after they have been discharged as relieved or cured in order that he may be informed of the recurrence of indigestion or other symptoms pointing to the return of the ulcer. Or if this is impossible because of change of residence or for other reasons the full importance of keeping in touch with a medical man should be explained to the patient. This paper deals particularly with the question of malignant disease of the abdominal viscera, but the fact that carcinoma may grow upon the base of a chronic gastric ulcer makes the consideration of treatment of ulcer important in this connection.

Welch's table of the cardinal symptoms of gastric carcinoma and gastric ulcer is given for reference.

Welch's Table. GASTRIC CANCER.

1. Tumor present in three fourths of cases.

2. Rare under forty years.

3 . Average duration about one year; rarely over two years.

4. Gastric hemorrhage frequent but rarely profuse; most common in the cachectic state.

5. Vomiting often has the pecuilarities of that of dilatation of the stomach.

6. Free HCl usually absent; lactic acid present.

7. Cancer fragments in washings or in vomitus. 